\title{
Retraction Note: Bioinformatic Analysis of Codon Usage and Phylogenetic Relationships in Different Genotypes of the Hepatitis C
}

\section{Virus}

\author{
Seyed Moayed Alavian ${ }^{1, *}$ \\ ${ }^{1}$ Professor of GI \& Hepatology, MELD Center, Tehran, Iran \\ "Corresponding author: Professor of GI \& Hepatology, MELD Center, Tehran, Iran. Email: alavian@thc.ir
}

Received 2020 January 31; Accepted 2020 January 31.

This retracts the article "Retracted Article: Bioinformatic Analysis of Codon Usage and Phylogenetic Relationships in Different Genotypes of the Hepatitis C Virus".

Following the publication of the corrigendum in issue 19(11) (http://hepatmon.com/en/articles/99558.html), the two recently added authors contacted the journal editor and claimed that the roles and order of authors of the published article (link: http://hepatmon.com/en/articles/15664.html) still needs to be changed.

As we already stated in the corrigendum, adding those two new authors was performed due to the official and definite decision made by the Ethics Department of Legal Medicine Organization of Iran and approval of Iran National Committee for Ethics in Biomedical Research (http://ethics.research.ac.ir/) (published in http://hepatmon.com/en/articles/99558.html). After receiving a new allegation raised on 8th Dec 2019 by the two recently added authors (Mohammad Fazel Soltani and Morteza Hadizadeh) that claimed some sort of ambiguity and uncertainty in the author's contribution and roles of the mentioned article, EIC and Publisher decided to re-evaluate their decision.

In a subsequent lengthy consultation with the publisher's ethical and legal teams, EIC reached the conclusion that the paper must be 'retracted' from the journal's record because of troubled and suspicious authorship problems both proved in the primary ethical committee (published in http://hepatmon.com/en/articles/99558.html) and final ethical committee (EIC and Publisher). Despite the authors' agreement to change the authors' contribution and roles in the mentioned article (date: 17 Dec 2019), we made our final decision to retract this paper due to authorship malpractice. However, the authors will be able to submit their paper elsewhere to reconstruct the public record of their study, without the regrettable circumstances that followed the publication of their article in Hepatitis Monthly.

All authors were informed of the retraction and retraction notice in advance of publication (31 Dec 2019).

Seyed Moayed Alavian

Editor-in-Chief 